\title{
SU(N) Coherent States and Irreducible Schwinger Bosons
}

\author{
Manu Mathur 1 and Indrakshi Raychowdhury 2 \\ S. N. Bose National Centre for Basic Sciences \\ JD Block, Sector III, Salt Lake City, Calcutta 700098, India
}

\begin{abstract}
We exploit the SU(N) irreducible Schwinger boson to construct $\mathrm{SU}(\mathrm{N})$ coherent states. This construction of $\mathrm{SU}(\mathrm{N})$ coherent state is analogous to the construction of the simplest Heisenberg-Weyl coherent states. The coherent states belonging to irreducible representations of $\mathrm{SU}(\mathrm{N})$ are labeled by the eigenvalues of the $(N-1)$ $\mathrm{SU}(\mathrm{N})$ Casimir operators and are characterized by $(N-1)$ complex orthonormal vectors describing the $\mathrm{SU}(\mathrm{N})$ group manifold.
\end{abstract}

\section{Introduction}

The concept of coherent states was introduced by Schrödinger [1] in the context of a harmonic oscillator. These harmonic oscillator coherent states, also called canonical coherent states, have been widely used in physics [2, 3, 4, 5, 6]. The next important coherent states are spin coherent states or SU(2) coherent states which are associated with angular momentum or the SU(2) group. Like canonical coherent states, they too have found wide applications in different branches of physics such as quantum optics, statistical mechanics, nuclear physics and condensed matter physics [2, 5, 6, 7, 8]. It is known that these spin coherent states can also be constructed using harmonic oscillators by exploiting either the HolsteinPrimakov or the Schwinger boson representation of the SU(2) Lie algebra [9, 10, 11]. This harmonic oscillator formulation of spin coherent states is appealing because of its simplicity as this construction is analogous to the simplest and oldest canonical coherent state construction. Further, unlike the standard construction of coherent states [6], this method does not require any knowledge of group representations or group elements and their actions on a particular weight vector, leading to many technical simplifications (see section 【I). Infact, these SU(2) coherent states in terms of harmonic oscillators have been implicitly contained in the seminal work of Schwinger [9] way back in 1952. The aim of the present work is to show that the above simple, uniform and explicit construction of canonical and spin or $\mathrm{SU}(2)$ coherent states can also be easily extended to all higher $\mathrm{SU}(\mathrm{N})$ groups. This is in contrast to the standard construction of $\mathrm{SU}(\mathrm{N})$ coherent states (i.e., by applying the $\mathrm{SU}(\mathrm{N})$ group elements on a particular weight vector) which is known to become more and more tedious as $\mathrm{N}$ increases [12, 13]. In the past, this problem has led to various different approaches [12, 13, 14, 15, 16, 17, 18] to construct SU(3) and SU(N) coherent states. In [12] $\mathrm{SU}(\mathrm{N})$ coherent states belonging to $\mathrm{SU}(\mathrm{N})$ symmetric representations are constructed by using fixed order polynomials of complex N-plets. In [13] a very special characterization of SU(N) group elements is exploited to construct $\mathrm{SU}(\mathrm{N})$ coherent states. In [14, 15, 16] Schwinger boson representation of SU(N) Lie algebra is used to construct $\mathrm{SU}(\mathrm{N})$ coherent states. In [17] the Schwinger oscillator representation of $\mathrm{SU}(3)$

\footnotetext{
${ }^{1}$ manu@bose.res.in

2indrakshi@bose.res.in
} 
coherent states is analyzed to discuss its relationship with the standard harmonic oscillator coherent states. In [18] SU(3) coherent states are constructed using a special parametrization of SU(3) group elements.

The present work exploits the $\mathrm{SU}(\mathrm{N})$ irreducible Schwinger bosons [19, 20] to construct $\mathrm{SU}(\mathrm{N})$ coherent states belonging to an arbitrary irreducible representation of $\mathrm{SU}(\mathrm{N})$. By definition, the $\mathrm{SU}(\mathrm{N})$ irreducible Schwinger bosons creation operators carry all the symmetries of $\mathrm{SU}(\mathrm{N})$ irreducible representations (see section $\amalg$ and IV). As a result all SU(N) irreducible representation states are monomials (not polynomials) of SU(N) irreducible Schwinger boson creation operators acting on the corresponding vacuum states. Therefore, as in the case of canonical and spin coherent states, they are the natural candidates for constructing the $\mathrm{SU}(\mathrm{N})$ coherent states. These coherent states are defined on the $\mathrm{SU}(\mathrm{N})$ group manifold characterized by an orthonormal set of $(N-1)$ complex $\mathrm{SU}(\mathrm{N})$ vectors. In addition, the $\mathrm{SU}(\mathrm{N})$ coherent states are labeled by the integer eigenvalues of $(N-1)$ Casimir operators which are the $(N-1)$ types of Schwinger boson number operators (see sections [II and IV).

The organization of the paper is as follows. We start with a very brief discussion on harmonic oscillator or canonical coherent states because the $\mathrm{SU}(\mathrm{N})$ coherent state construction in this work is analogous to this simplest construction. In section $\amalg$ we illustrate this similarity by constructing SU(2) coherent states in terms of a doublet of harmonic oscillators or equivalently $\mathrm{SU}(2)$ Schwinger bosons. As mentioned earlier, this construction has been exploited by Schwinger [9] to compute SU(2) recoupling coefficients. At the end of section $\llbracket$ the simplifications obtained by this Schwinger boson approach to coherent states over the standard approach of applying a SU(2) group element on a particular weight vector are highlighted. In sections III and IV we further extend the above $\mathrm{SU}(2)$ construction to $\mathrm{SU}(3)$ and $\mathrm{SU}(\mathrm{N})$ respectively. These $\mathrm{SU}(3)$ and then $\mathrm{SU}(\mathrm{N})$ extensions of $\mathrm{SU}(2)$ coherent states are again trivial as they correspond to:

- replacing $\mathrm{SU}(2)$ Schwinger boson doublet by $(N-1) \mathrm{SU}(\mathrm{N})$ irreducible Schwinger bosons N-plets,

- replacing $\mathrm{SU}(2)$ group manifold (i.e., a doublet of complex numbers) by $\mathrm{SU}(\mathrm{N})$ group manifold (i.e., $N-1$ N-plets of complex numbers).

The section $\amalg$ on $\mathrm{SU}(3)$ is added to make the transition from $\mathrm{SU}(2)$ (section【) to $\mathrm{SU}(\mathrm{N})$ (section IV) easy.

In the simplest example of the Heisenberg-Weyl group, the Lie algebra contains three generators. It is defined in terms of creation annihilation operators $\left(a, a^{\dagger}\right)$ satisfying

$$
\left[a, a^{\dagger}\right]=\mathcal{I}, \quad[a, \mathcal{I}]=0, \quad\left[a^{\dagger}, \mathcal{I}\right]=0 .
$$

This algebra has only one infinite dimensional unitary irreducible representation. The states within this representation are the occupation number states $|n\rangle \equiv \frac{\left(a^{\dagger}\right)^{n}}{\sqrt{n !}}|0\rangle$ with $n=0,1,2 \ldots$. The coherent states of the Heisenberg-Weyl group are defined over a complex manifold as:

$$
|z\rangle_{[\infty]}=\exp \left(z a^{\dagger}\right)|0\rangle=\sum_{n=0}^{\infty} F_{n}(z)|n\rangle .
$$

In (2) the subscript $[\infty]$ on the coherent states is the irreducible representation index. It implies that these coherent states are defined over the infinite dimensional irreducible representation of the group. The sum in (2) runs over all the basis vectors $|n\rangle$ belonging to this infinite dimensional representation. The coefficients:

$$
F_{n}(z)=\frac{z^{n}}{\sqrt{n !}}
$$


are the coherent state expansion coefficients which are analytic functions of the group manifold coordinate $\mathrm{z}$. The resolution of identity property of the coherent state (2) follows from the group transformation property. Let us define the operator: $O_{[\infty]} \equiv \int e^{-|z|^{2}} d z d \bar{z}|z\rangle_{[\infty]}[\infty]\langle z|$. Under the Heisenberg Weyl group element [6] $g_{\mathrm{h} w} \equiv \exp \left(i \alpha+w a-\bar{w} a^{\dagger}\right):|z\rangle_{[\infty]} \rightarrow e^{i \alpha+z w-\frac{w w}{2}}|z-\bar{w}\rangle_{[\infty]}$. It is trivial to see that the operator $O_{[\infty]}$ defined above is invariant under $g_{\mathrm{h} w}$. Therefore, by Schurs lemma it is proportional to unity operator.

The purpose of this work is to generalize (2), (3) for Heisenberg-Weyl group to SU(N) for arbitrary N (see (10), (12) for SU(2); (30), (32) for SU(3) and (47), (49) for SU(N)). We start with SU(2) construction [9, 10] first.

\section{II $S U(2)$ Coherent States}

The Heisenberg Weyl coherent state construction can be readily generalized to the simplest compact group $\mathrm{SU}(2)$ by utilizing the Schwinger representation of SU(2) Lie algebra: $\left[J^{\mathrm{a}}, J^{\mathrm{b}}\right]=i \epsilon^{\mathrm{abc}} J^{\mathrm{c}}$. We define [9]:

$$
J^{\mathrm{a}} \equiv \frac{1}{2} a_{\alpha}^{\dagger}\left(\sigma^{\mathrm{a}}\right)_{\beta}^{\alpha} a^{\beta} .
$$

In (4) $\sigma^{\mathrm{a}}$ with a $=1,2,3$ denote the three Pauli matrices. The doublet of harmonic oscillator creation and annihilation operators $a^{\alpha}$ and $a_{\alpha}^{\dagger}$ or equivalently Schwinger bosons in (4) satisfy the simple bosonic commutation relations $\left[a^{\alpha}, a_{\beta}^{\dagger}\right]=\delta^{\alpha}{ }_{\beta}$ with $\alpha, \beta=1,2$. The vacuum state $|0,0\rangle$ of these two oscillators will be denoted by $|0\rangle$. Under $\mathrm{SU}(2)$ transformations the Schwinger boson creation operators transform as doublets:

$$
a_{\alpha}^{\dagger} \rightarrow a_{\beta}^{\dagger}\left(\exp i \theta^{\mathrm{a}} \frac{\sigma^{\mathrm{a}}}{2}\right)_{\alpha}^{\beta} \text {. }
$$

The defining equations (4) imply that the $\mathrm{SU}(2)$ Casimir operator is simply the total number operator:

$$
C \equiv \sum_{\alpha=1}^{2} a_{\alpha}^{\dagger} a^{\alpha} \equiv a^{\dagger} \cdot a .
$$

The eigenvalues of $C$ will be denoted by $n$. The various states in the irreducible representation $n(=2 j)$ are:

$$
\left|\alpha_{1} \alpha_{2} \ldots . \alpha_{n}\right\rangle_{[n]} \equiv a_{\alpha_{1}}^{\dagger} a_{\alpha_{2}}^{\dagger} \ldots . . a_{\alpha_{n}}^{\dagger}|0\rangle
$$

The corresponding SU(2) Young tableau is shown in Figure (1). Note that the state in (7) is invariant under all $n$ ! permutations of the $\mathrm{SU}(2)$ indices $\alpha_{1}, \alpha_{2}, \cdots, \alpha_{n}$. This is because all $\mathrm{SU}(2)$ creation operators on the right hand side of (7) commute amongst themselves. In other words, the SU(2) Schwinger boson creation operators carry the the symmetries of the SU(2) Young tableau $\sqrt[3]{ }$ which is shown in Figure (1). Therefore, the $(n+1)$ states in (7) belong to $\mathrm{SU}(2)$ irreducible representation with total angular momentum $j=\frac{n}{2}$.

The SU(2) group manifold $S^{3}$ can also be described by a doublet of of complex numbers $\left(z^{1}, z^{2}\right)$ of unit magnitude:

$$
|z|^{2} \equiv\left|z_{1}\right|^{2}+\left|z_{2}\right|^{2}=1
$$

This is because any $\mathrm{SU}(2)$ matrix $\mathcal{U}_{2}$ can be written as:

$$
\mathcal{U}_{2}=\left(\begin{array}{cc}
z_{1} & z_{2} \\
-z_{2}^{*} & z_{1}
\end{array}\right)
$$

\footnotetext{
${ }^{3}$ This obvious symmetry argument will not be true for higher SU(N) (sections III and IV leading to the definition of SU(N) irreducible Schwinger bosons. In terms of SU(N) irreducible Schwinger bosons the SU(N) irreducible states will be monomials like 7 .
} 


\begin{tabular}{|l|l|l|l|}
\hline$a_{\alpha_{1}}^{+}$ & $a_{\alpha_{2}}^{+}$ & $\ldots \ldots .$. & $a_{\alpha_{\mathrm{n}}}^{+}$ \\
\hline
\end{tabular}

$\mathrm{n}$ boxes

Figure 1: $\mathrm{SU}(2)$ Young table in the $n=2 j$ representation. The monomial state (7) carries the horizontal permutation symmetries of this Young tableau.

with $\mathcal{U}_{2}^{+} \mathcal{U}_{2}=\mathcal{U}_{2} \mathcal{U}_{2}^{+}=1,\left|\mathcal{U}_{2}\right|=1$. At this stage one can trivially combine the $\mathrm{SU}(2)$ irreducible states in (7) and the $\mathrm{SU}(2)$ group manifold coordinates in (8) to construct the generating function of the SU(2) coherent states:

$$
|z\rangle \equiv\left|z^{1}, z^{2}\right\rangle=\exp \left(z \cdot a^{\dagger}\right)|0\rangle=\sum_{n=0}^{\infty} \frac{\left(z \cdot a^{\dagger}\right)^{n}}{n !}|0\rangle=\sum_{n=0}^{\infty}|z\rangle_{n} .
$$

Above $z \cdot a^{\dagger} \equiv z^{1} a_{1}^{\dagger}+z^{2} a_{2}^{\dagger}$ and $|z\rangle_{[n]}$ is the coherent state in the $\mathrm{SU}(2)$ representation $j=n / 2$ :

$$
|z\rangle_{[n]}=\sum_{\alpha_{1}, \alpha_{2}, \cdots, \alpha_{n}=1}^{2} F^{\alpha_{1} \alpha_{2} \ldots \alpha_{n}}(z) a_{\alpha_{1}}^{\dagger} a_{\alpha_{2}}^{\dagger} \cdots a_{\alpha_{n}}^{\dagger}|0\rangle=\sum_{\alpha_{1}, \alpha_{2}, \cdots, \alpha_{n}=1}^{2} F^{\alpha_{1} \alpha_{2} \ldots \alpha_{n}}(z) \underbrace{\left|\alpha_{1} \alpha_{2} \cdots \alpha_{n=2 j}\right\rangle_{[j]}}_{\text {Su(2) irrep. } j=\frac{n}{2}}
$$

Like in Heisenberg Weyl case (3), the SU(2) coherent state structure functions in the irreducible representation $j=\frac{n}{2}$ are:

$$
F^{\alpha_{1} \alpha_{2} \ldots \alpha_{n}}\left(z^{1}, z^{2}\right) \equiv \frac{1}{n !} z^{\alpha_{1}} z^{\alpha_{2}} \ldots z^{\alpha_{n}}
$$

Note that they are analytic functions of group manifold coordinates. The resolution of identity property again follows from the group transformation laws. The coherent state structure in (10) and the the SU(2) transformations (5) imply that under group transformations: $\left|z^{1}, z^{2}\right\rangle_{[n]} \rightarrow\left|z^{\prime 1}, z^{\prime 2}\right\rangle_{[n]}$ where $\left(z^{\prime 1}, z^{\prime 2}\right)$ are the SU(2) rotated coherent state co-ordinates:

$$
z^{\prime \alpha}=\left(\exp i\left(\theta^{\mathrm{a}} \frac{\sigma^{\mathrm{a}}}{2}\right)\right)_{\beta}^{\alpha} z^{\beta}
$$

Therefore, under the SU(2)transformations the coherent states $|z\rangle \equiv\left|z_{1}, z_{2}\right\rangle$ transform amongst themselves on $S^{3}$ as the constraint (8) remains invariant under (13). Again we define the operator:

$$
O_{[n]} \equiv \int d \mu(z)\left(|z\rangle_{[n][n]}\langle z|\right)=\int d^{2} z^{1} d^{2} z^{2} \delta\left(\left|z^{1}\right|^{2}+\left|z^{2}\right|^{2}-1\right)|z\rangle_{[n][n]}\langle z| .
$$

The operator $O_{[n]}$ is invariant under all SU(2) transformations of the coherent states $|z\rangle_{[n]}$. Therefore,

$$
\left[Q^{\mathrm{a}}, \mathcal{O}_{[n]}\right]=0, \quad \forall \mathrm{a}=1,2, \ldots, 8
$$

The Schur's Lemma implies that $O_{[n]}$ is proportional to identity operator. Before generalizing (10) to SU(N), it is illustrative to briefly mention the standard group theoretical coherent state construction procedure [6]. We characterize the $\mathrm{SU}(2)$ group elements $\mathrm{U}$ by the Euler angles, i.e, $U(\theta, \phi, \psi) \equiv \exp -i \phi J_{3} \exp -i \theta J_{2} \exp -i \psi J_{3}$. The $\mathrm{SU}(2)$ coherent states are constructed as:

$$
|\theta, \phi, \psi\rangle_{j}=U(\theta, \phi, \psi)|j, j\rangle,=\sum_{m=-j}^{+j} C_{m}(\theta, \phi, \psi)|j, m\rangle,
$$


The coefficients $C_{m}(\theta, \phi, \psi)$ are given by,

$$
C_{m}(\theta, \phi, \psi)=e^{-i(m \phi+j \psi)}\left[\frac{2 j !}{(j+m) !(j-m) !}\right]^{\frac{1}{2}}\left[\sin \frac{\theta}{2}\right]^{j-m}\left[\cos \frac{\theta}{2}\right]^{j+m} .
$$

It is clear that the corresponding construction is difficult for higher $\mathrm{SU}(\mathrm{N})$ group as we need to know all the $\mathrm{SU}(\mathrm{N})$ representations, Euler angles and the group elements to implement this procedure. On the other hand, the coherent states in (10) are straightforward generalization of the Heisenberg-Weyl coherent states in (2) and bypass all the problems mentioned above. Our aim in this work is to further extend this simple coherent state construction to $\mathrm{SU}(\mathrm{N})$ with arbitrary $\mathrm{N}$. As we will see the only new input required for this purpose is the replacement of $S U(2)$ Schwinger bosons by $S U(N)$ irreducible Schwinger bosons [19, 20]. We first deal with $\mathrm{SU}(3)$ group in detail.

\section{SU(3) Coherent States}

We start with a brief review of SU(3) irreducible Schwinger bosons [19, 20] and then construct SU(3) coherent states.

\section{III.1 The Irreducible Schwinger Boson Representations of SU(3)}

As the rank of $\mathrm{SU}(3)$ group is two, we need two independent triplets to construct any arbitrary irrep of $\mathrm{SU}(3)$. We take them to be two independent harmonic oscillator triplets or equivalently Schwinger boson and denote them by: $a_{\alpha}^{\dagger}[1]$ and $a_{\alpha}^{\dagger}[2]$. The SU(3) generators in terms of these Schwinger bosons are:

$$
Q^{\mathrm{a}}=a^{\dagger}[1] \frac{\lambda^{\mathrm{a}}}{2} a[1]+a^{\dagger}[2] \frac{\lambda^{\mathrm{a}}}{2} a[2], a=1,2, \cdots, 8 .
$$

In (16) $\lambda^{\text {a's }}$ are the Gell-Mann matrices. Under SU(3) transformations both types of Schwinger boson creation operators transform as triplets:

$$
a_{\alpha}^{\dagger}[i] \rightarrow a_{\beta}^{\dagger}[i]\left(\exp i \theta^{\mathrm{a}} \frac{\lambda^{\mathrm{a}}}{2}\right)_{\alpha}^{\beta} \quad i=1,2 .
$$

The defining equations (16) immediately imply that the two SU(3) Casimirs commuting with all the generators are the two total number operators:

$$
C[1] \equiv \mathcal{N}_{1} \equiv a^{\dagger}[1] \cdot a[1], \quad C_{2} \equiv \mathcal{N}_{2} \equiv a^{\dagger}[2] \cdot a[2] .
$$

It is obvious that $\left[C[i], Q^{a}\right]=0, \forall i=1,2 ; a=1,2, \cdots, 8$ as each $Q^{a}$ contains one creation and one annihilation operator of either type [i=1] or [i=2]. Their eigenvalues are denoted by $n_{1}$ and $n_{2}$ respectively. The corresponding irreducible representation with $n_{1} \geq n_{2}$ is denoted 4 by $\left[n_{1}, n_{2}\right]$. The associated SU(3) Young tableau is shown in Figure (2).

The monomial states constructed out of the two SU(3) fundamental Schwinger bosons:

$$
\left|\alpha_{1} \alpha_{2} \cdots \alpha_{n_{1}} ; \beta_{1} \beta_{2} \cdots \beta_{n_{2}}\right\rangle \equiv\left(a_{\alpha_{1}}^{\dagger}[1] a_{\alpha_{2}}^{\dagger}[1] \ldots . a_{\alpha_{n_{1}}}^{\dagger}[1]\right)\left(a_{\beta_{1}}^{\dagger}[2] a_{\beta_{2}}^{\dagger}[2] \ldots . a_{\beta_{n_{2}}}^{\dagger}[2]\right)|0\rangle
$$

\footnotetext{
${ }^{4}$ Note that $n_{1}$ and $n_{2}$ are the numbers of two $\mathrm{SU}(3)$ triplets and not triplets and anti-triplets. As an example, $[1,1]$ represents the anti-triplet $3^{*}$ representation.
} 


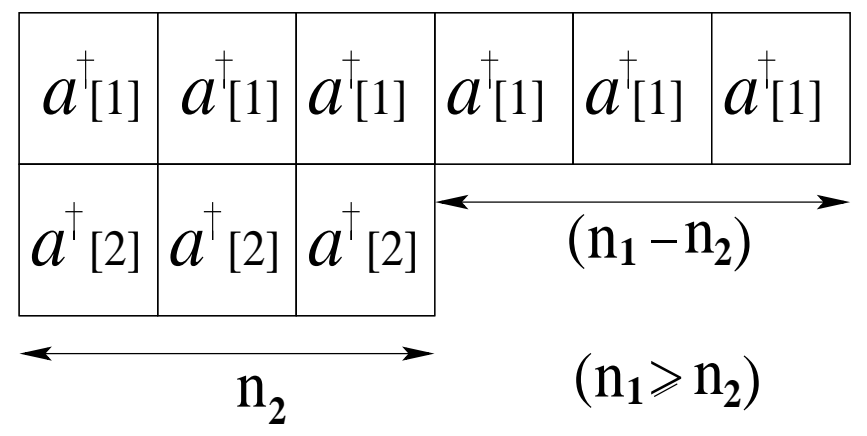

Figure 2: SU(3) Young table in the $\left[n_{1}, n_{2}\right]$ representation. The monomial state (27) in terms of SU(3) irreducible Schwinger bosons and not (19) carries all the symmetries of this SU(3) Young tableau.

are eigenstates of $C$ [1] and $C$ [2] with eigenvalues $n_{1}$ and $n_{2}$ respectively. However, unlike $\mathrm{SU}(2)$ case where the monomial states (7) are SU(2) irreducible, the corresponding monomial states (19) are SU(3) reducible and do not form the SU(3) irreducible representation $\left[n_{1}, n_{2}\right]$. Like in SU(2) case, the monomial state (19) carries the horizontal symmetries of $\mathrm{SU}(3)$ Young tableaux as the Schwinger bosons creation operators on the right hand side commute amongst themselves. However, the vertical antisymmetry needs to be imposed to get SU(3) irreducibility. We achieve this by imposing the following constraint [20, 21]:

$$
\hat{L}_{12} \equiv a^{\dagger}[1] \cdot a[2]=0
$$

on the monomial states (19). As an example, the simple $\left[n_{1}=1, n_{2}=1\right]$ irreducible representation of $\mathrm{SU}(3)$ corresponds to the anti-triplet $3^{*}$ and the corresponding states are given by:

$$
|\alpha ; \beta\rangle_{\left[n_{1}=1, n_{2}=1\right]}=-|\beta ; \alpha\rangle_{\left[n_{1}=1, n_{2}=1\right]} \equiv\left(a_{\alpha}^{\dagger}[1] a_{\beta}^{\dagger}[2]-a_{\beta}^{\dagger}[1] a_{\alpha}^{\dagger}[2]\right)|0\rangle .
$$

The three states in (21) trivially satisfy the constraint (20) as they are anti-symmetric. Therefore, they belong to $\mathrm{SU}(3)$ irreducible representation $3^{*}$. In recent works [20, 19] we have defined SU(3) irreducible Schwinger bosons $A^{\dagger}[i]$ with $i=1,2$ as:

$$
\begin{aligned}
& A_{\alpha}^{\dagger}[1]=a_{\alpha}^{\dagger}[1] \\
& A_{\alpha}^{\dagger}[2]=a_{\alpha}^{\dagger}[2]-\frac{1}{\mathcal{N}_{1}-\mathcal{N}_{2}+2}\left(a^{\dagger}[2] \cdot a[1]\right) a_{\alpha}^{\dagger}[1]
\end{aligned}
$$

The SU(3) irreducible Schwinger bosons in (23) are constructed such that [19, 20]:

$$
\begin{aligned}
& {\left[\left(a^{\dagger}[1] \cdot a[2]\right), A^{+\alpha}[1]\right] \simeq 0} \\
& {\left[\left(a^{\dagger}[1] \cdot a[2]\right), A^{\dagger \beta}[2]\right] \simeq 0}
\end{aligned}
$$

Where, ' $\simeq 0$ ' means that the commutators are weakly zero. In other words, the above commutators annihilate all SU(3) irreducible states satisfying (20). The irreducible Schwinger bosons further satisfy:

$$
\left[A_{\alpha}^{\dagger}[1], A_{\beta}^{\dagger}[1]\right]=0, \quad\left[A_{\alpha}^{\dagger}[2], A_{\beta}^{\dagger}[2]\right]=0 .
$$

The defining equations (??) imply that their transformation properties are exactly same as (33):

$$
A_{\alpha}^{\dagger}[i] \rightarrow A_{\beta}^{\dagger}[i]\left(\exp i \theta^{\mathrm{a}} \frac{\lambda^{\mathrm{a}}}{2}\right)_{\alpha}^{\beta} \quad i=1,2 .
$$


We now consider the most general monomial state constructed out of SU(3) irreducible Schwinger bosons:

$$
\left|\alpha_{1} \alpha_{2} \ldots \alpha_{n_{1}} ; \beta_{1} \beta_{2} \ldots \beta_{n_{2}}\right\rangle_{\left[n_{1} n_{2}\right]} \equiv\left(A_{\beta_{1}}^{\dagger}[2] A_{\beta_{2}}^{\dagger}[2] \ldots A_{\beta_{n_{2}}}^{\dagger}[2]\right)\left(A_{\alpha_{1}}^{\dagger}[1] A_{\alpha_{2}}^{\dagger}[1] \ldots A_{\alpha_{n_{1}}}^{\dagger}[1]\right)|0\rangle .
$$

This monomial state directly creates the SU(3) Young tableau with $n_{1}$ and $n_{2}$ boxes in the first and second rows respectively. This is because the inbuilt constraint (20) ensures the vertical antisymmetry and the commutators (25) ensure the horizontal symmetries of SU(3) Young tableau. Therefore, the monomial states in (27) belong to $\left[n_{1}, n_{2}\right]$ irreducible representation of $\mathrm{SU}(3)$. We now exploit this simple fact to further extend the definition of Heisenberg Weyl, SU(2) coherent states (2) and (10) to SU(3) group.

\section{III.2 Construction of SU(3) Coherent States}

Similar to SU(2) case (8) and (9), the eight dimensional SU(3) group manifold can be characterized by two complex triplets: $z_{\alpha}[1]$ and $z_{\alpha}[2](\alpha=1,2,3)$ which satisfy the orthonormality constraints:

$$
\bar{z}[1] \cdot z[1]=1=\bar{z}[2] \cdot z[2], \quad \bar{z}[1] \cdot z[2]=0 .
$$

This is because any $\mathrm{SU}(3)$ matrix $\mathcal{U}_{3}$ can be written as:

$$
\mathcal{U}_{3}=\left(\begin{array}{lll}
z_{1}[1] & z_{1}[2] & (\bar{z}[1] \wedge \bar{z}[2])_{1} \\
z_{2}[1] & z_{2}[2] & (\bar{z}[1] \wedge \bar{z}[2])_{2} \\
z_{3}[1] & z_{3}[2] & (\bar{z}[1] \wedge \bar{z}[2])_{3}
\end{array}\right)
$$

with $\mathcal{U}_{3} \mathcal{U}_{3}^{+}=\mathcal{U}_{3}^{+} \mathcal{U}_{3}=1$ and $\operatorname{det}\left(\mathcal{U}_{3}\right)=\left|\mathcal{U}_{3}\right|=1$ due to the orthonormality constraints (28).

We define the $\mathrm{SU}(3)$ coherent states generating function as:

$$
|z[1], z[2]\rangle \equiv \exp \left(z[2] \cdot A^{\dagger}[2]\right) \exp \left(z[1] \cdot A^{\dagger}[1]\right)|0\rangle
$$

Note that this construction is $\mathrm{SU}(3)$ extension of $\mathrm{SU}(2)$ coherent state generating function (10). We can project $\mathrm{SU}(3)$ coherent state in the representation $\left[n_{1}, n_{2}\right]$ by considering the corresponding term in the generating function (30):

$$
\begin{aligned}
& |z[1], z[2]\rangle_{\left[n_{1}, n_{2}\right]} \equiv \frac{\left(z[2] \cdot A^{\dagger}[2]\right)^{n_{2}}}{n_{2} !} \frac{\left(z[1] \cdot A^{\dagger}[1]\right)^{n_{1}}}{n_{1} !}|0\rangle \\
& =\sum_{\alpha_{1} . . \alpha_{n_{1}}=1}^{3} \sum_{\beta_{1} . . \beta_{n_{2}}=1}^{3} F^{\alpha_{1} . . \alpha_{n_{1}} ; \beta_{1} . . \beta_{n_{2}}}(z[1], z[2]) \underbrace{\left|\alpha_{1} \alpha_{2} \ldots \alpha_{n_{1}} ; \beta_{1} \beta_{2} \ldots \beta_{n_{2}}\right\rangle_{\left[n_{1} n_{2}\right]}}_{\text {Su(3) irrep. }\left[n_{1}, n_{2}\right]} .
\end{aligned}
$$

In (31) the SU(3) coherent state structure functions,

$$
F^{\alpha_{1} . \alpha_{n_{1}} ; \beta_{1} . \beta_{n_{2}}}(z[1], z[2])=\frac{1}{n_{1} ! n_{2} !} z[1]^{\alpha_{1}} z[1]^{\alpha_{2}} \ldots z[1]^{\alpha_{n_{1}}} z[2]^{\beta_{1}} z[2]^{\beta_{2}} \ldots z[2]^{\beta_{n_{2}}} .
$$

are analytic functions of SU(3) group manifold co-ordinates. Like in SU(2) case, the resolution of identity property follows from the group transformation laws. Using the $\mathrm{SU}(3)$ transformations (26), we find that the $\mathrm{SU}(3)$ coherent states transform as: $|z[1], z[2]\rangle_{\left[n_{1}, n_{2}\right]} \rightarrow\left|z^{\prime}[1], z^{\prime}[2]\right\rangle_{\left[n_{1}, n_{2}\right]}$ where

$$
z^{\prime \alpha}[1]=\left(\exp i\left(\theta^{\mathrm{a}} \frac{\lambda^{\mathrm{a}}}{2}\right)\right)_{\beta}^{\alpha} z^{\beta}[1], \quad z^{\prime \alpha}[2]=\left(\exp i\left(\theta^{\mathrm{a}} \frac{\lambda^{\mathrm{a}}}{2}\right)\right)_{\beta}^{\alpha} z^{\beta}[2] .
$$


Again like in $\mathrm{SU}(2)$ case, $z[1]$ \& $z$ [2] transform like $\mathrm{SU}(3)$ triplets, the orthonormality conditions (28) remains invariant under the $\mathrm{SU}(3)$ transformations. In other words, the coherent state (48) defined at a point $(z[1], z[2])$ transform to the coherent state at $\left(z^{\prime}[1], z^{\prime}[2]\right)$ on the $\mathrm{SU}(3)$ group manifold. Therefore, the operator $O_{\left[n_{1}, n_{2}\right]}$ :

$$
O_{\left[n_{1}, n_{2}\right]} \equiv \int d \mu(z)\left(|z[1], z[2]\rangle_{\left[n_{1}, n_{2}\right] \quad\left[n_{1}, n_{2}\right]}\langle z[1], z[2]|\right)
$$

with SU(3) Haar measure

$$
\int d \mu(z) \equiv\left(\int d^{2} z[1] d^{2} z[2]\right)\left(\prod_{\alpha, \beta=1}^{2} \delta\left(z[\alpha] \cdot z^{*}[\beta]-\delta_{\alpha, \beta}\right)\right.
$$

is invariant under all $\mathrm{SU}(3)$ transformations (33):

$$
\left[Q^{\mathrm{a}}, O_{\left[n_{1}, n_{2}\right]}\right]=0, \quad \forall \mathrm{a}=1,2, \ldots ., 8
$$

Therefore, by Schur's Lemma $O_{\left[n_{1}, n_{2}\right]}$ is proportional to identity operator.

The SU(3) coherent states (31) and the structure functions (32) are straightforward generalization of the SU(2) coherent states (10) and the corresponding structure functions (12) respectively. The latter, in turn, are SU(2) generalization of the oldest Heisenberg-Weyl or harmonic oscillator coherent states (2) and the associated structure functions (3).

\section{SU(N) coherent state}

Like the previous section on $\mathrm{SU}(3)$, this section has two parts. In the first part we briefly describe the $\mathrm{SU}(\mathrm{N})$ irreducible Schwinger bosons [20] and in the second part we exploit it to define $\mathrm{SU}(\mathrm{N})$ coherent states.

\section{IV.1 Irreducible Schwinger Boson Representations of SU(N)}

The rank of $\mathrm{SU}(\mathrm{N})$ group is $(N-1)$. Therefore, the fundamental constituents required to construct any arbitrary irrep. of $\mathrm{SU}(\mathrm{N})$ can be chosen to be $N-1$ independent Schwinger boson $N$-plets: $a^{\dagger \alpha}[1], a^{\dagger \alpha}[2], a^{\dagger \alpha}[3], \ldots, a^{\dagger \alpha}[N-1]$ with $\alpha=1,2,3, . ., N$. The $\mathrm{SU}(\mathrm{N})$ generators in terms of these Schwinger bosons are:

$$
Q^{\mathrm{a}}=\sum_{i=1}^{N-1} a^{\dagger}[i] \frac{\Lambda^{\mathrm{a}}}{2} a[i], \quad \mathrm{a}=1,2, \cdots,\left(N^{2}-1\right) .
$$

Above $\Lambda^{\text {a's }}$ are the generalization of Gell-Mann matrices for $\mathrm{SU}(\mathrm{N})$. The $N(N-1)$ Harmonic oscillators present in (36) creates a $N(N-1)$ dimensional Hilbert space $\mathcal{H}_{\mathrm{HO}}^{\mathrm{N}(\mathrm{N}-1)}$. The $\mathrm{SU}(\mathrm{N})$ transformations are:

$$
a_{\alpha}^{\dagger}[i] \rightarrow a_{\beta}^{\dagger}[i]\left(\exp i \theta^{\mathrm{a}} \frac{\lambda^{\mathrm{a}}}{2}\right)_{\alpha}^{\beta} \quad i=1,2, \cdots,(N-1) .
$$

The defining equations (36) imply that the $(N-1)$ Casimirs associated with $\mathrm{SU}(\mathrm{N})$ group, denoted by $C_{i}$, are the $(N-1)$ number operators

$$
\mathcal{N}_{i} \equiv C_{i}=a^{\dagger}[i] \cdot a[i], \quad i=1,2, . .,(N-1)
$$




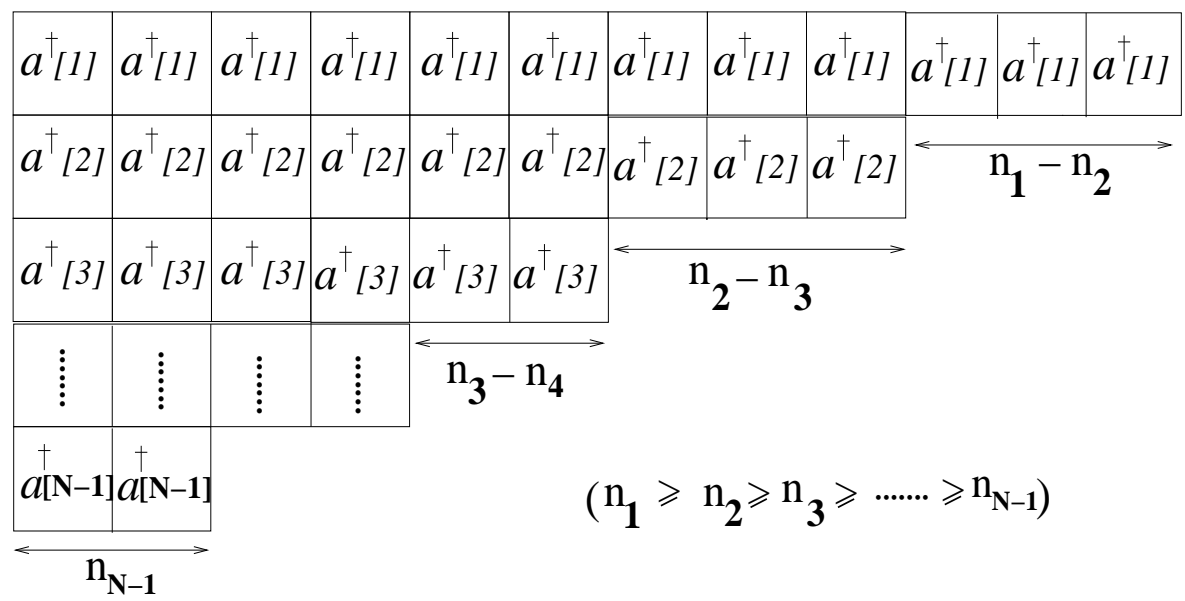

Figure 3: $\mathrm{SU}(\mathrm{N})$ Young table in the $\left[n_{1}, n_{2}, n_{3}, \ldots, n_{N-1}\right]$ representation. The $\mathrm{SU}(\mathrm{N})$ irreducible Schwinger boson monomial state (44) and not (39) carries all the symmetries of this SU(N) Young tableau.

A particular $\mathrm{SU}(\mathrm{N})$ irreducible representation is labeled by their eigenvalues: $\left[n_{1}, n_{2}, \ldots, n_{N-1}\right]$. The $\mathrm{SU}(\mathrm{N})$ monomial eigenstate:

$$
\left|\alpha_{1}^{[1]} . . \alpha_{n_{1}}^{[1]} ; \alpha_{1}^{[2]} . . \alpha_{n_{2}}^{[2]} ; \cdots ; \alpha_{1}^{[N-1]} . . \alpha_{n_{N-1}}^{[N-1]}\right\rangle \equiv \underbrace{a_{\alpha_{1}^{[1]}}^{\dagger}[1] . . a_{\alpha_{n_{1}}^{[1]}}^{\dagger}[1]}_{n_{1} \text { of } a^{+}[1] \ldots \ldots .} \cdots \underbrace{a_{\alpha_{1}^{[N-1]}}^{\dagger}[N-1] . . a_{\alpha_{n_{N-1}}^{\dagger N-1]}}^{\dagger}[N-1]}_{\ldots \ldots . n_{N-1} \text { of } a^{+}[N-1]}|0\rangle,
$$

satisfies

$$
C_{i}\left|\alpha_{1}^{[1]} . . \alpha_{n_{1}}^{[1]} ; \alpha_{1}^{[2]} . . \alpha_{n_{2}}^{[2]} ; . . ; \alpha_{1}^{[N-1]} . . \alpha_{n_{N-1}}^{[N-1]}\right\rangle=n_{i}\left|\alpha_{1}^{[1]} . . \alpha_{n_{1}}^{[1]} ; \alpha_{1}^{[2]} . . \alpha_{n_{2}}^{[2]} ; . . ; \alpha_{1}^{[N-1]} . . \alpha_{n_{N-1}}^{[N-1]}\right\rangle .
$$

However, like in $\mathrm{SU}(3)$ case (19), these states are $\mathrm{SU}(\mathrm{N})$ reducible due to the presence of $\mathrm{SU}(\mathrm{N})$ invariants. These invariants can be removed by implementing the symmetries of the associated SU(N) Young tableau in Figure 3 which contains $n_{i}$ boxes in the $i^{\text {th }}$ row.

Like in SU(3) case we impose the following $\frac{(N-1)(N-2)}{2}$ constraints [21, 20]:

$$
\hat{L}_{i j}=a^{\dagger}[i] \cdot a[j] \approx 0, \quad i, j=1,2, \cdots(N-1) \text { and } i<j .
$$

on the monomial states (39). Like in SU(3) case, we can trivialize all the constraints in (40) in terms of SU(N) irreducible Schwinger bosons [20]. The SU(N) irreducible Schwinger boson creation operators acting on the vacuum directly create states having all the symmetries of the corresponding Young tableaux. These new irreducible Schwinger bosons $A^{\dagger}[i]$ for $i=1,2, . ., N-1$ are related to the ordinary Schwinger bosons in (36) as follows:

$$
A^{\dagger \alpha}[k]=a^{\dagger \alpha}[k]+\sum_{r=1}^{k-1} \sum_{\left\{i_{1}, \ldots, i_{r}\right\}=1}^{k-1} F_{i_{1}}^{k} F_{i_{2}}^{k} \cdots F_{i_{r}}^{k} \hat{L}_{k i_{1}} \hat{L}_{i_{1} i_{2}} \ldots \hat{L}_{i_{r-1} i_{r}} a^{\dagger \alpha}\left[i_{r}\right] .
$$

In (41) $k=1,2, \cdots(N-1)$ and the prime over the second summation $\left(\sum^{\prime}\right)$ implies that the ordering $k>i_{1}>i_{2}>$ $\ldots>i_{r}$ has to be maintained. The general form of $F_{i}^{k}\left(n_{1}, . ., n_{N-1}\right)$ is given by,

$$
F_{i}^{k}=-\frac{1}{n_{i}-n_{k}+1+k-i} .
$$


The defining equations (41) imply that the SU(N) irreducible Schwinger bosons also transform as SU(N) N-plets:

$$
A_{\alpha}^{\dagger}[i] \rightarrow A_{\beta}^{\dagger}[i]\left(\exp i \theta^{\mathrm{a}} \frac{\lambda^{\mathrm{a}}}{2}\right)_{\alpha}^{\beta} \quad i=1,2, \cdots,(N-1) .
$$

Therefore, as in SU(2) case, the Hilbert space created by the monomials of SU(N) irreducible Schwinger boson (41) creation operators is isomorphic to the space of irreducible representations of $S U(N)$. In other words the state

$$
\begin{aligned}
& \left|\alpha_{1}^{[1]}, \alpha_{2}^{[1]}, \ldots, \alpha_{n_{1}}^{[1]} ; \alpha_{1}^{[2]}, \alpha_{2}^{[2]}, \ldots, \alpha_{n_{2}}^{[2]}, \ldots, \alpha_{1}^{[N-1]}, \alpha_{2}^{[N-1]} \ldots, \alpha_{n_{N-1}}^{[N-1]}\right\rangle_{\left[n_{1}, n_{2}, \cdots n_{N-1}\right]}
\end{aligned}
$$

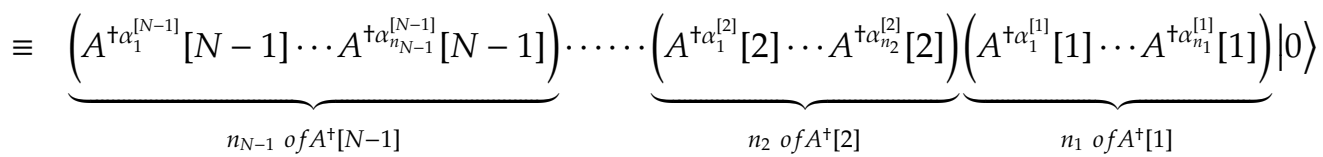

carries all the symmetries of an $\mathrm{SU}(\mathrm{N})$ Young tableaux shown in Figure 3 .

\section{IV.2 Construction of SU(N) Coherent States}

Like in SU(2) and SU(3) cases in (8) and (28) respectively, we characterize the SU(N) group manifold by $N-1$ number of complex $N$-plets: $\left\{z_{\alpha}[i]\right\}, i=1,2, \ldots, N-1$ and $\alpha=1,2, \ldots N$ following orthonormality constraints:

$$
\bar{z}[\alpha] \cdot z[\beta]=\delta_{\alpha, \beta} .
$$

With the above parametrization any $\mathrm{SU}(\mathrm{N})$ matrix has the following form:

$$
\mathcal{U}_{N}=\left(\begin{array}{cccccc}
z_{1}[1] & z_{1}[2] & \ldots & \ldots & z_{1}[N-1] & (\bar{z}[1] \wedge \bar{z}[2] \wedge \ldots \wedge \bar{z}[N-1])_{1} \\
z_{2}[1] & z_{2}[2] & \ldots & \ldots & z_{2}[N-1] & (\bar{z}[1] \wedge \bar{z}[2] \wedge \ldots \wedge \bar{z}[N-1])_{2} \\
\vdots & \vdots & \ddots & & \vdots & \vdots \\
\vdots & \vdots & & \ddots & \vdots & \vdots \\
z_{N}[1] & z_{N}[2] & \ldots & \ldots & z_{N}[N-1] & (\bar{z}[1] \wedge \bar{z}[2] \wedge \ldots \wedge \bar{z}[N-1])_{N}
\end{array}\right)
$$

At this stage we generalize (11) and (31) to define the $\mathrm{SU}(\mathrm{N})$ coherent state generating function as:

$$
|z[1], z[2], \ldots z[N-1]\rangle \equiv \exp \left(z[N-1] \cdot A^{\dagger}[N-1]\right) \ldots \ldots \exp \left(z[2] \cdot A^{\dagger}[2]\right) \exp \left(z[1] \cdot A^{\dagger}[1]\right)|0\rangle .
$$

Note that the coherent state generating function (47) contains all possible irreducible representations of SU(N). Further, the expressions for $\mathrm{SU}(\mathrm{N}+1)$ and $\mathrm{SU}(\mathrm{N})$ coherent states differ only by the last exponential factor in (47). Therefore, the present $\mathrm{SU}(\mathrm{N})$ coherent state construction is iterative in nature. Now, projecting out a specific coherent state denoted by the set of particular values of the $\mathrm{SU}(\mathrm{N})$ Casimirs, i.e., $A^{+}[i] \cdot A[i]$ having eigenvalue $n_{i}$ with $i=1,2, \cdots(N-1)$ and $n_{1} \geq n_{2} \geq \ldots \geq n_{N-1}$ we get the $\mathrm{SU}(\mathrm{N})$ coherent state in the irreducible representation $\left[n_{1}, n_{2}, \cdots, n_{N-1}\right]$ :

$$
\begin{aligned}
& |z[1], z[2], \ldots z[N-1]\rangle_{\left[n_{1}, n_{2} \cdots n_{N-1}\right]} \equiv \frac{\left(z[N-1] \cdot A^{\dagger}[N-1]\right)^{n_{N-1}}}{n_{N-1} !} \ldots \frac{\left(z[2] \cdot A^{\dagger}[2]\right)^{n_{2}}}{n_{2} !} \frac{\left(z[1] \cdot A^{\dagger}[1]\right)^{n_{1}}}{n_{1} !}|0\rangle \\
= & \sum_{\alpha_{1}^{[1]} \ldots, \alpha_{n_{1}}^{[1]}=1}^{N} \sum_{\alpha_{1}^{[2]} \ldots, \alpha_{n_{2}}^{[2]}=1}^{N} \sum_{\alpha_{1}^{[N-1]}, \ldots, \alpha_{n_{N-1}}^{[N-1]}=1}^{N} F^{\alpha_{1}^{[1]} \ldots \alpha_{n_{1}}^{[1]} \ldots \alpha_{1}^{[N-1]} \ldots \alpha_{n_{N-1}}^{[N-1]}(z[1], z[2] \cdots z[N-1])} \underbrace{\left|\alpha_{1}^{[1]} . \alpha_{n_{1}}^{[1]} \cdots \alpha_{1}^{[N-1]} . . \alpha_{n_{N-1}}^{[N-1]}\right\rangle_{\left[n_{1}, n_{1} \cdot n_{N-1}\right]}}_{\text {SU(N) irrep. state }[4]}
\end{aligned}
$$


In (48) the $\mathrm{SU}(\mathrm{N})$ coherent state structure functions are given by:

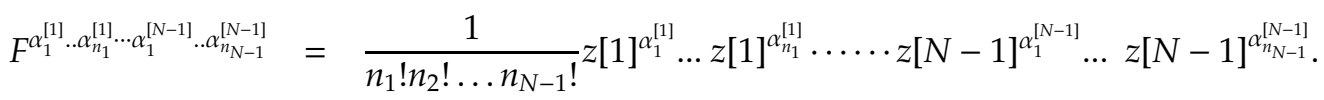

The states in (48) depend smoothly on the SU(N) group manifold coordinates. We now check the resolution of identity. Like in the previous $\mathrm{SU}(2)$ and $\mathrm{SU}(3)$ sections, under $\mathrm{SU}(\mathrm{N})$ transformations (43) all the $(N-1)$ coherent state co-ordinates $z[i]$ transform as N-plets:

$$
z_{\alpha}[i] \rightarrow z_{\alpha}^{\prime}[i]=z_{\beta}[i]\left(\exp i \sum_{\mathrm{a}=1}^{N^{2}-1} \theta^{\mathrm{a}} \Lambda^{\mathrm{a}}\right)_{\alpha}^{\beta} .
$$

We again define the operator $O_{\left[n_{1}, n_{2}, \cdots n_{N-1}\right]}$ as:

$$
O_{\left[n_{1}, n_{2}, \cdots, n_{N-1}\right]} \equiv \int d \mu(z)\left(|z[1], z[2], \ldots z[N-1]\rangle_{\left[n_{1}, n_{2}, \cdots n_{N-1}\right]}{ }_{\left[n_{1}, n_{2}, \cdots n_{N-1}\right]}\langle z[1], z[2], \ldots z[N-1]|\right) .
$$

In (51) $\int d \mu(z)$ is the $\mathrm{SU}(\mathrm{N})$ invariant Haar measure:

$$
\int d \mu(z) \equiv\left[\prod_{\alpha=1}^{N-1} \int d^{2} z[\alpha]\right] \prod_{\alpha, \beta} \delta\left(z[\alpha] \cdot z^{*}[\beta]-\delta_{\alpha, \beta}\right) .
$$

Under $\mathrm{SU}(\mathrm{N})$ transformations $(50), O_{[N]}$ remains invariant. Therefore,

$$
\left[Q^{\mathrm{a}}, \mathcal{O}_{\left[n_{1}, n_{2}, \cdots, n_{N-1}\right]}\right]=0, \quad \forall \mathrm{a}=1,2, \ldots . . N^{2}-1 .
$$

The Schur's Lemma implies:

$$
\mathcal{O}_{\left[n_{1}, n_{2}, \cdots, n_{N-1}\right]}=I_{\left[n_{1}, n_{2}, \cdots, n_{N-1}\right]} .
$$

In (53) $I_{\left[n_{1}, n_{2}, \cdots, n_{N-1}\right]}$ is proportional to identity operator in the irreducible representation subspace. We again emphasize that the $\mathrm{SU}(\mathrm{N})$ coherent states in (48) are the most straightforward extension of the Heisenberg Weyl, SU(2) and SU(3) coherent states in (2), (11) and (31) respectively.

\section{Conclusions}

We have exploited SU(N) irreducible Schwinger boson creation operators to construct $\mathrm{SU}(\mathrm{N})$ coherent states. This construction is analogous to the simplest and the oldest harmonic oscillator coherent state construction. This procedure is iterative in N. It is also self contained as it does not require any prior knowledge of SU(N) group elements and their representations. This novel $\mathrm{SU}(\mathrm{N})$ coherent state construction can be used to compute $\mathrm{SU}(\mathrm{N})$ Clebsch-Gordan and recoupling coefficients. This amounts to generalizing the Schwinger method to compute these coefficients for $\mathrm{SU}(2)$ (see section 3 of [9] on the addition of angular momenta) to $\mathrm{SU}(\mathrm{N})$ ). This is particularly interesting as these $\mathrm{SU}(\mathrm{N})$ coupling coefficients for arbitrary $\mathrm{N}$ are not yet known in closed form. The work in this direction is in progress and will be reported elsewhere. 


\section{References}

[1] E. Schrödinger, Naturwissenshafter, 14, 644, (1926).

[2] Klauder J R and Skagerstam B-S 1985 Coherent States: Applications in Physics and Mathematical Physics (Singapore: World Scientic),

Inomata A, Kuratsuji H and Gerry C C 1992 Path Integrals and Coherent States of SU $(2)$ and SU $(1,1)$ (Singapore: World Scientic).

[3] R. J. Glauber, Phys. Rev. 130 (1963) 2529.

[4] E. C. G. Sudarshan, Phys. Rev. Lett. 10 277. Phys. Rev. 131 (1963) 2766.

[5] W. M. Zhang, D. H. Feng and R. Gilmore, "Coherent states: Theory and some Applications," Rev. Mod. Phys. 62, 867 (1990).

[6] Perelomov A 1986 Generalized Coherent States and Their Applications (New York: Springer), Perelomov A 1977 Sov. Phys.Usp. 20 703,

A. M. Perelomov, Commun. Math. Phys. 26, 222 (1972).

[7] A. Auerbach, Interacting Electrons and Quantum Magnetism , (Springer-Verlag, N.Y.), A. Auerbach and D.P. Arovas, in Field Theories In Condensed Matter Physics, Ed. Z. Tesanovich, Addison-Wesley (1990).

[8] A. Klein E. R. Marshalek, Rev. Mod. Phys. 63, 375-557 (1991).

[9] Schwinger J 1952 Atomic Energy Commission Report No NYO-3071, Mattis D 1982 The Theory of Magnetism (New York: Harper and Row).

[10] Radcliffe J M 1971 J. Phys. A: Gen. Phys. 4313

[11] Eriksson K-E and Skagerstam B-S 1979 J. Phys. A: Math. Gen. 122175

[12] Gitman D M and Shelepin A 1993 J. Phys. A: Math. Gen. 26313.

[13] Kae Nemoto, J. Phys. A 33 (2000) 3493.

[14] Manu Mathur, Diptiman Sen, J. Math. Phys. 42 (2001) 4181.

[15] M. Mathur and H. S. Mani, J. Math. Phys. 43, 5351 (2002) [arXiv:quant-ph/0206005].

[16] R. R. Puri, Phys. Rev. A 50, (1994) 5309.

[17] S. Chaturvedi, N. Mukunda, J. Math. Phys. 43 (2002) 5278.

[18] S. Gnutzmann and M. Kus, J. Phys. A 31, (1998) 9871.

[19] Ramesh Anishetty, Manu Mathur and Indrakshi Raychowdhury, J. Math. Phys. 50, 053503 (2009) |arXiv:0901.0644 [math-ph]].

[20] Manu Mathur, Indrakshi Raychowdhury and Ramesh Anishetty, arXiv:1003.5487 [math-ph] (to be published in J. Math. Phys.).

[21] M. Moshinsky, Rev. Mod. Phys. 34813 (1962), J. Math. Phys. 4, 1128 (1963). 